\title{
Planungsrechnungen zur Optimierung von Zuchtplänen der Rasse Merinofleischschaf
}

\section{Summary} Title of the paper: Alternative selection strategies for the Mutton Merino breed to optimize breeding
systems

This paper deals with the investigation of alternative selection strategies for the Mutton Merino breed to improve lamb meat production and fertility. A main topic is the application of ultrasonic scanning to evaluate the meat content on live animals. The aim of model calculations was the optimisation and comparison of five different selection strategies within three different schemes of gene dissimation. First a basic scheme was defined and optimised (selection on field records, one-step selection, no scanning). It was regarded as the reference scheme to which all other breeding systems were related. The following schemes considered both, selection with and without scanning. A second scheme, also based on field records, includes two-step selection of rams (called improved field test scheme). In a third and fourth scheme a level of uniform environment was assumed for ram progeny testing. These are a central testing station on the one hand with slaughtering and carcass evaluation on progeny, and one or more contract farms on the other (without slaughtering). For a fifth selection scheme an open nucleus was assumed with ram progeny testing in associated test herds.

In a deterministic approach using the ZPLAN computer program, the monetary genetic gain for the breeding objective (traits lambs weaned, litter weight at weaning, postweaning daily gain and lean meat content) and the profit were calculated for each scheme after optimisation of various biological-technical coefficients. The highest profit was achieved with a nucleus scheme (DM 9,16). Due to low recording costs the basic scheme was second (DM 7,19) and, because of high costs, the station scheme was last (DM 4,22). The other two schemes were intermediate (DM 6,98 for the scheme with contract farms and DM 6,58 for the improved field test scheme). On an average over all schemes, scanning resulted in a 0,24 DM (i.e. almost 30 per cent) increase of the monetary genetic gain and a threefold higher genetic gain for lean meat percentage. In all schemes scanning lead also to a higher profit because the higher return of selection based on scanning exceeded the higher costs.

Key words: breeding systems, breeding costs, genetic improvement, mutton merino breed

\section{Zusammenfassung}

Für die Rasse Merinofleischschaf wurden Zuchtsysteme untersucht, mit denen die Mast- und Schlachtleistung sowie die Fruchtbarkeit verbessert werden können. Schwerpunkt ist die Verwendung der Ultraschallmeßmethode zur Bestimmung der Schlachtleistung am lebenden Tier. Die Planungsrechnungen umfassen die Untersuchung von drei Zuchtsystemen mit Optimierung von fünf verschiedenen Zuchtplänen. Es wurde zuerst eine Ausgangssituation definiert und optimiert. Diese Ausgangssituation war Bezugspunkt für die Ergebnisse aller anderen Zuchtpläne. In einem zweiten Zuchtsystem wurde die Wirksamkeit der Leistungsprüfung untersucht, wenn eine Prüfstation in verschiedenen Zuchtebenen integriert wird und in den Zuchtplänen verschiedene Selektionsstrategien verfolgt werden. Ausgehend von der Optimalvariante wurde ein drittes Zuchtsystem geprüft, für das ein Nukleus charakteristisch ist. Die Optimierung erfolgte mit dem Programm ZPLAN auf der Basis der Genfluß-Methode. In einem deterministischen Ansatz wurden unter Berücksichtigung verschiedener Populationsparameter sowie der Züchtungskosten über die Genflußmethode der Züchtungsgewinn je Mutterschaf bzw. der monetäre Gesamtzuchtfortschritt je Mutterschaf und Jahr ermittelt. Allen Zuchtplänen wurde ein einheitliches Zuchtziel mit folgenden Merkmalen und Grenznutzen unterstellt: Aufgezogene Lämmer je Mutter und Ablammung (86,71 DM/Lamm), Summe der Absetzgewichte der Lämmer (5,56 DM/kg), Tägliche Zunahme vom Absetzen bis zum Mastende (0,06 DM/g), Fleischanteil im Schlachtkörper (1,17 DM/\%). 
Im Ergebnis der Optimierungen ist der höchste Züchtungsgewinn mit 9,16 DM bei einem Zuchtsystem mit Nukleus zu erzielen. Bei Verwendung von Ultraschall in der Leistungsprüfung folgen die Varianten „Vertragsbetriebe“" (Leistungsprüfung unter einheitlichen Umweltbedingungen; keine Prüfstation) mit 6,98 DM, "Feldprüfung“ (keine Prüfstation) mit 6,58 DM. Bedingt durch hohe Züchtungskosten schneiden Zuchtpläne mit einer Prüfstation in der Leistungsprüfung mit 4,22 DM vergleichsweise schlecht ab. Zuchtpläne mit Ultraschall haben cinen im Mittel um 0,24 DM höheren monetären jährlichen Gesamtzuchtfortschritt. Die Verwendung von Ultraschall in der Leistungsprifung führt im Mittel aller Zuchtpläne zu einem dreifach so hohen jährlichen naturalen Zuchtfortschritt im Merkmal Fleischanteil $(0,55 \%)$ im Vergleich zu Zuchtplănen ohne Ultraschall. Die Züchtungskosten für Zuchtpläne mit Ultraschall liegen ca. 0,30 DM über denen ohne Ultraschallmessungen, allerdings sind die Züchtungsgewinne bei Verwendung von Ultraschall bedingt durch die höheren Züchtungserträge größer.

Schlüsselwörter: Zuchtpläne, Zuchtkosten, Zuchtfortschritt, Merinofleischschaf

Das Merinofleischschaf wurde in den letzten vier Jahrzehnten in den neuen Bundesländern als Zweinutzungsrasse gezüchtet. Im Mittelpunkt der Zuchtarbeit standen die Verbesserung der Wollqualität und die Erhöhung der Wollmenge je Tier. Heute erfordert das Preisgefüge die Betonung der Fleischleistung. Das Merinofleischschaf in Reinzucht zu einer weiteren Fleischschafrasse umzuzüchten, erscheint angesichts der vorhandenen leistungsfähigen Fleischschafrassen nicht notwendig. Vielmehr sollte die gute Fruchtbarkeit sowie eine besondere Eigenschaft des Merinofleischschafes - seine Asaisonalität - genutzt werden. Die Ausrichtung auf ein neues Produktionsziel erfordert ein neues Zuchtziel. Der Rasseausschuß „Merinofleischschafe“ beschloß daher am 19.09.92 ein für die Merinofleischschafe (Ost) und Merinofleischschafe (West) einheitliches Zuchtziel. Wie schnell das Zuchtziel umgesetzt werden kann, ist wesentlich von einem effizienten Zuchtprogramm abhängig.

Im folgenden Beitrag wird daher anhand verschiedener Zuchtpläne dargestellt, welche Auswirkungen sich auf den Zuchtfortschritt und Züchtungsgewinn ergeben, wenn verschiedene Alternativen der Selektion und Leistungsprüfung angewandt werden und die Ultraschallmeßmethode am lebenden Tier als Hilfsmittel zur Bestimmung des Fleischund Fettanteils im Schlachtkörper eingefuhrt wird.

\section{1 .}

\section{Material und Methode}

In einem Zuchtplan wird auf der Grundlage genetischer Parameter und ökonomischer Faktoren ein Modell beschrieben, das alle notwendigen züchterischen Maßnahmen beinhaltet, um einen hohen züchterischen Nutzen erzielen zu können. Ein Zuchtplan umfaßt neben dem Zuchtziel die Beschreibung der Zuchtebenen und Selektionsgruppen, der Selektionsintensität, der Art und Weise der Leistungsprüfung sowie des Generationsintervalls. Für die Bewertung eines Zuchtplanes können verschiedene Kriterien herangezogen werden. In der Regel sind es der naturale Zuchtfortschritt für Einzelmerkmale und der Züchtungsgewinn, der sich aus den Maßnahmen einer Selektionsrunde in einem vorgegebenen Investitionszeitraum ergibt. Dieser errechnet sich aus der Differenz zwischen Züchtungsertrag und Züchtungskosten bei vorgegebener Verzinsung. 
Die Planungsrechnungen wurden mit dem Programm ZPLAN (KARRAS u.a., 1994) durchgeführt. Methodisch fließen in das Programm die Selektionstheorie, die Indextheorie mit der Zweistufenselektion, die Genflußmethode und Grundzüge der Investitionstheorie ein (HILL, 1974; ELSEN und MOCQUOT, 1974; WEBER, 1976; BRASCAMP, 1978). Das Programm wird für die Optimierung von Zuchtplänen verwendet, wobei die Optimierung nicht nach mathematischen Optimierungsfunktionen erfolgt. Statt dessen wird die bestmögliche Kombination der Populationsparameter hinsichtlich der Maximierung des Zuchtfortschritts bzw. des Züchtungsgewinns gesucht. Die Ergebnisse werden in DM ausgewiesen, wobei der Züchtungsgewinn, der Züchtungsertrag und die Züchtungskosten auf ein Mutterschaf im Investitionszeitraum bezogen sind, während der Bezugspunkt des monetären Gesamtzuchtfortschritts ein Mutterschaf und Jahr darstellt.

Zur Aufstellung und Berechnung eines Zuchtplanes sind eine Reihe von Modellannahmen notwendig. Diese umfassen das Zuchtziel und die ökonomischen Wichtungsfaktoren der Einzelmerkmale, Züchtungskosten, genetische Parameter sowie biologische und technische Koeffizienten.

Allen Zuchtplänen wurde ein einheitliches Zuchtziel unterstellt, deren Merkmale wie folgt ökonomisch gewichtet wurden (MÜLLER, 1995):

- Aufgezogene Lämmer je Mutter und Ablammung

- Summe der Absetzgewichte der Lämmer

- Tägliche Zunahme vom Absetzen bis zum Mastende

- Fleischanteil im Schlachtkörper

86,71 DM/Lamm, $5,56 \mathrm{DM} / \mathrm{kg}$, $0,06 \mathrm{DM} / \mathrm{g}$, $1,17 \mathrm{DM} / \%$.

In den Planungsrechnungen wurden konstante Züchtungsparameter und -kosten unterstellt, die den Unterlagen zweier Schafzuchtverbände entnommen wurden. Züchtungskosten sind Kosten, die über die Produktionskosten hinaus für die Durchführung von Zuchtmaßnahmen erforderlich sind (Tab. 1).

Tabelle 1 Fixe und variable Züchtungskosten in der Merinofleischschafzucht (Fix and variable breeding costs in the
breeding programme for Merinoland)

\begin{tabular}{|c|c|c|}
\hline Fixe Züchtungskosten in [DM/Jahr] & 5000 Tiere & 20000 Tiere \\
\hline Büro & 20000 & 40000 \\
\hline Büroausstattung & 3500 & 7000 \\
\hline Dienstwagen & 3500 & 7000 \\
\hline Ultraschallgerät & \multirow{2}{*}{\multicolumn{2}{|c|}{$\begin{array}{r}2000^{11} \\
54000^{11}\end{array}$}} \\
\hline Prüfstation & & \\
\hline Sonstiges & 1000 & 2000 \\
\hline
\end{tabular}

\begin{tabular}{lrr}
\multicolumn{2}{l}{ Variable Züchtungskosten in [DM/Jahr] } \\
Herdbuchführung & [je Tier] & \\
Ultraschallmessung & [je Messung] & 0,40 \\
ELP/NKP-Station & [je Tier] & 1,50 \\
ELP-Feld & [je Tier] & 25,00 \\
\hline ELP-Eigenleistungspruffung; NKP - Nachkommenprüfung; " - jâthrliche Abschreibung & 1,00 \\
\hline
\end{tabular}

Die Aufwendungen für Büromiete, Büroausstattung, Dienstwagen, Prüfstation und Ultraschallgerät werden den fixen Züchtungskosten zugeordnet. Diese Kosten sind in ei- 
nem weiten Rahmen unabhängig von der zu prüfenden Tierzahl, sobald man sich für ein bestimmtes Prüfverfahren entschieden hat. Beispielsweise ist bei der Eigenleistungsprüfung mit Ultraschall nur ein Gerät erforderlich, gleichgültig, ob damit die Nachkommen von 5000 oder 20000 Muttertieren gemessen werden. Kosten, die sich aus den allgemeinen Aufgaben eines Zuchtverbandes ergeben (z. B. Auktion, Körung oder Geschäftsführung), werden nicht mit einbezogen, da das keine Züchtungskosten im engeren Sinne sind. Bei den fixen Züchtungskosten wird von einem Minimum von 5000 weiblichen Zuchttieren in der Population ausgegangen. Weiterhin wird angenommen, daß sich diese Werte verdoppeln, wenn mehr als 20000 Zuchttiere betreut werden müssen. Das gilt nicht für das Ultraschallgerät und die Prüfstation. Dafür ist eine Neuanschaffung bzw. Erweiterung notwendig, wenn die Kapazitäten erschöpft sind, und die sind abhängig von der zu prüfenden Tierzahl. Die variablen Züchtungskosten umfassen die Reise- und Lohnkosten sowie Verbrauchsmaterial und Sonstiges. Diese Kostengrößen werden in Tabelle 1 auf die einzelnen Tätigkeitskomplexe verteilt. Sowohl Ertrags- als auch Aufwandskomponenten können zu unterschiedlichen Zeitpunkten anfallen. Um sie vergleichen zu können, müssen sie auf einen Bezugszeitpunkt abgezinst werden. Daran anlehnend werden für die Planungsrechnungen folgende Annahmen gemacht:
Kalkulationszinssatz für den Aufwand
$4 \%$
Kalkulationszinssatz für den Ertrag
$6 \%$
Investitionsdauer
20 Jahre.

Tabelle 2

Biologisch-technische Koeffizienten (Biological and technical coefficients)

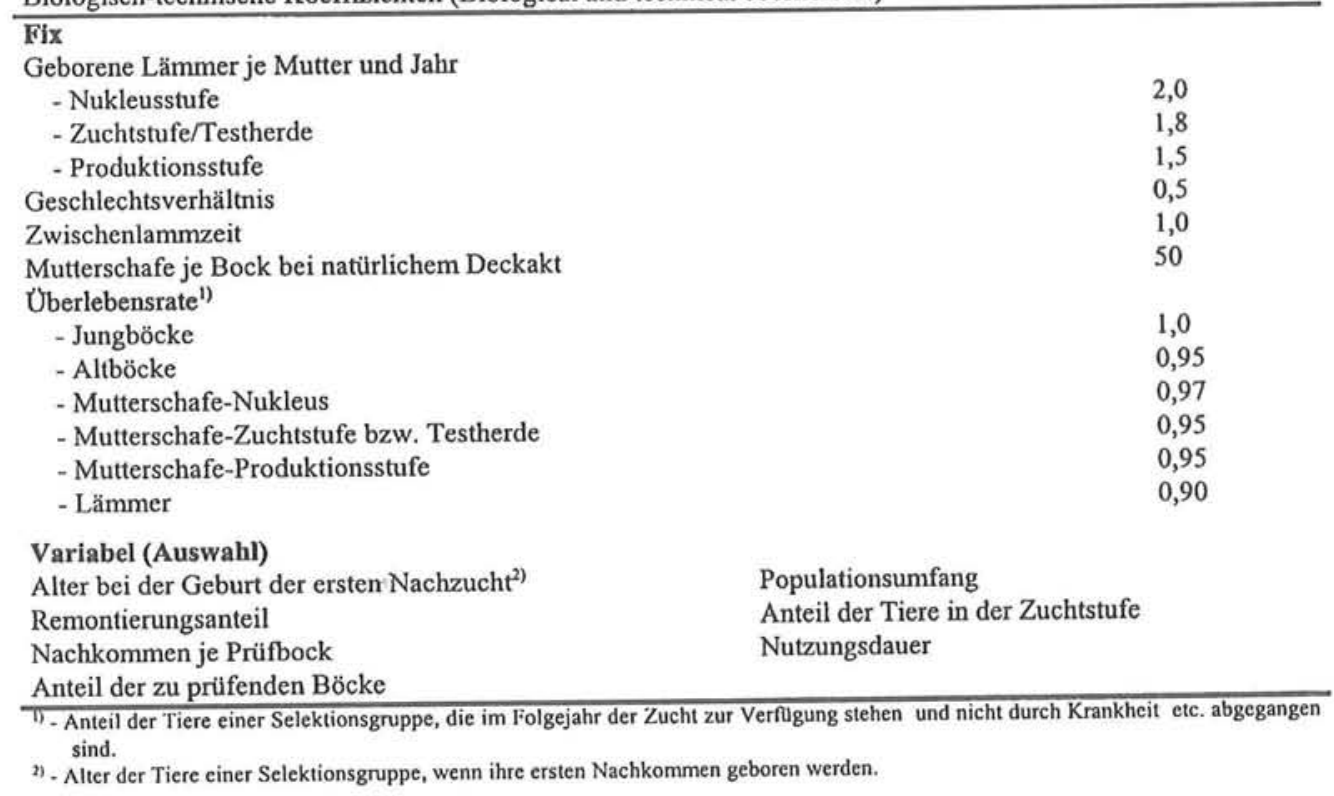

Für die Beschreibung des Zuchtablaufes müssen relevante biologische und technische 
Koeffizienten (Zuchtparameter) vorgegeben werden (Tab. 2). Es wird zwischen fixen und variablen Koeffizienten unterschieden. Die fixen Koeffizienten sind bei jedem Zuchtplan gleich, während die variablen Koeffizienten variiert werden, um die Veränderungen der Zielgrößen (z. B. Züchtungsgewinn) feststellen zu können.

Im vorliegenden Beitrag werden drei verschiedene Zuchtsysteme vorgestellt (Abb.). Innerhalb der Zuchtsysteme werden verschiedene Zuchtpläne definiert. Alle Zuchtpläne sind nach Züchtungsgewinn optimiert.

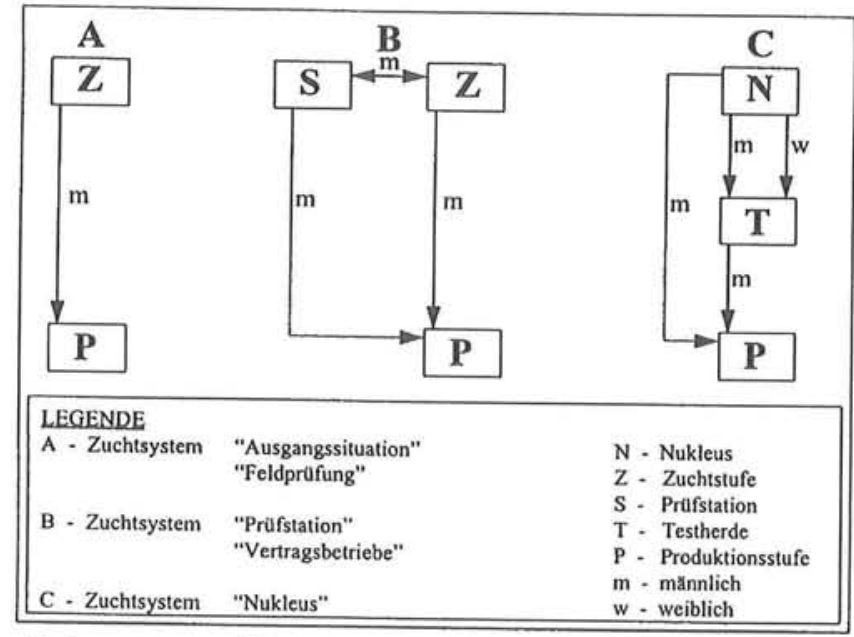

Abb.: Alternative Zuchtsysteme zur Übertragung des Zuchtfortschritts (in Anlehnung an BICHARD, 1971) (Alternative systems for disseminating the genetic improvement)

Zuchtsystem A. Das Zuchtsystem entspricht weitgehend dem in der züchterischen Praxis vorherrschenden Übertragungsmodell. Der Zuchtfortschritt wird über männliche Tiere aus der Zuchtstufe in die Produktionsstufe übertragen. Weibliche und männliche Tiere stehen, einmal in das Herdbuch aufgenommen, der Zucht uneingeschränkt zur Verfügung. Alle Mutterschafe sind potentielle Bockmütter. Für dieses Zuchtsystem wurden die Varianten „Ausgangssituation“ und „Feldprüfung“ untersucht. In der Variante „Ausgangssituation“ wird die Nachzucht einer Zuchtherde nur nach der Eigenleistung selektiert. Sie umfaßt die Merkmale tägliche Zunahme und die Bemuskelungsnote bei männlichen und weiblichen Tieren. Im Index werden die Eigenleistungen von Vater, Mutter und der Halbgeschwister berücksichtigt. Ultraschallmessungen werden in der Leistungsprüfung nicht angewendet. Mit der "Ausgangssituation“ soll der einfachste Zuchtplan als Ausgangsbasis dargestellt werden, mit dem alle anderen Zuchtpläne verglichen werden.

Die „Feldprüfung“ unterscheidet sich zur Ausgangssituation durch eine Zweistufenselektion im männlichen Pfad. Zusätzlich wird in diesem Zuchtplan der Einfluß der U1traschallmessungen auf den Züchtungsgewinn untersucht.

Zuchtsystem B. Mit diesem Zuchtsystem wird untersucht, wie sich die Prüfung unter einheitlichen Umweltbedingungen (Prüfstation bzw. Vertragsbetrieb) auf den Zucht- 
fortschritt und den Züchtungsgewinn auswirkt. Auch hier werden nachfolgend zwei Varianten (Zuchtplan „Prüfstation“ und Zuchtplan „Vertragsbetriebe") näher dargestellt. In beiden Varianten wird eine kombinierte Eigenleistungs- und Nachkommenprüfung durchgeführt. Im Zuchtplan „Prüfstation“ werden in der Nachkommenprüfung der Zuchtböcke die täglichen Zunahme und die Schlachtergebnisse (Benotung von Fettauflage und Muskelfülle am Schlachtkörper) ihrer Nachkommen berücksichtigt. Im Zuchtplan „Vertragsbetriebe“ wird auf eine Schlachtung verzichtet, die fixen Kosten sind im Vergleich zu einer zentralen Prüfstation geringer.

Zuchtsystem C. Für dieses Zuchtsystem ist ein Nukleus charakteristisch, in dem die besten Tiere zusammengefaßt sind. Die Jungböcke aus dem Nukleus werden an einer Testherde an Hand von Nachkommen geprüft. Aus der Testherde können die besten Mutterschafe in den Nukleus übernommen werden. Der Nukleus ist offen.

2 .

Ergebnisse

In Tabelle 3 werden die Ergebnisse der Planungsrechnungen für die Ausgangssituation beschrieben. Nach der Optimierung ist in diesem Zuchtplan mit einem Zuchtfortschritt von 0,0063 Lämmern zu rechnen. Werden alle naturalen Zuchtfortschritte mit dem entsprechenden ökonomischen Wichtungsfaktor multipliziert, ergibt das in der Summe über alle Zuchtzielmerkmale einen monetären Gesamtzuchtfortschritt von 1,08 DM je Mutterschaf und Jahr.

Mit Hilfe der Genflußmethode kann der Züchtungsertrag je Mutterschaf und Investitionsperiode ermittelt werden. Er ist abhängig von der Investitionsperiode und dem Beitrag der einzelnen Selektionsgruppen. Er beträgt für das Merkmal Aufgezogene Lämmer 4,92 DM. Die Summe über alle Merkmale ergibt einen Züchtungsertrag von 9,18 DM. Der Züchtungsgewinn ist die Differenz zwischen Züchtungsertrag und Züchtungskosten und beträgt in der Aussgangssituation 7,19 DM je Mutterschaf und Investitionsperiode.

Tabelle 3

Ergebnisse für die Zielgrößen nach Optimienung der Ausgangssituation (Results for various objective criteria after optimising the basic situation)

\begin{tabular}{ll}
\hline Naturaler Zuchtfortschritt & \\
Aufgezogene Lämmer & $0,0063 \mathrm{Lämmer}$ \\
Aufzuchtgewicht & $0,0167 \mathrm{~kg}$ \\
Masttagszunahme & $5,2900 \mathrm{~g}$ \\
Fleischanteil & $0,0966 \%$ \\
Monetärer Gesamtzuchtfortschritt & $1,08 \mathrm{DM}$ \\
Züchtungsertrag & $9,18 \mathrm{DM}$ \\
Züchtungskosten & $1,99 \mathrm{DM}$ \\
Züchtungsgewinn & $7,19 \mathrm{DM}$ \\
\hline
\end{tabular}

In den nachfolgenden Modellen wird die Leistungsprüfung einmal mit und eimal ohne Ultraschallmessungen durchgeführt. Die Feldprüfung unterscheidet sich zur Ausgangssituation durch die Einfuhrung einer Zweistufenselektion der männlichen Zuchttiere. Damit verbunden ist eine Nachkommenschaftsprüfung. Bedingt durch das längere Ge- 
nerationsintervall, sinkt der Züchtungsgewinn auf 6,58 DM, wenn Ultraschallmessungen in der Leistungsprüfung angewendet werden bzw. auf 4,01 DM ohne Ultraschallmessungen (Tab. 4).

Zuchtpläne mit einer in der Leistungsprüfung integrierten Prüfstation erzielen mit 10,03 DM den höchsten Züchtungsertrag. Bedingt durch die mit 5,81 DM bzw. 5,56 DM höchsten Züchtungskosten fällt der Züchtungsgewinn je Mutterschaf mit 4,22 DM bzw. 2,14 DM aber am niedrigsten aus.

Wird ein Zuchtplan um eine Testherde sowie einen Nukleus erweitert und eine Zweistufenselektion bei weiblichen und männlichen Zuchttieren durchgeführt, kann mit 13,01 DM und 9,16 DM sowohl der höchste Züchtungsertrag als auch der höchste Züchtungsgewinn aller untersuchten Alternativen erzielt werden.

In der Feldprüfung läßt sich mit einem optimierten Zuchtplan ein jährlicher monetärer Zuchtfortschritt von 1,00 DM je Mutterschaf erzielen (Tab. 4). Wird statt der Ultraschallmessungen eine einfache Bonitur zur Schätzung des Fleisch- und Fettgehaltes benutzt, sinkt der monetäre jährliche Zuchtfortschritt auf 0,74 DM. Die gleiche Aussage trifft für die Varianten „Prüfstation“ und „Vertragsbetriebe“ zu. Im Nukleus wurde eine Variante ohne Ultraschallmessungen nicht untersucht.

Tabelle 4

Vergleich von Zuchtplänen mit und ohne Ultraschall nach Züchtungsgewinn [DM] (Comparsion of breeding systems with an without ultrasonic scanning according to their profit)

\begin{tabular}{lccccc}
\hline $\begin{array}{c}\text { Zielgrößen } \\
\text { situation }\end{array}$ & $\begin{array}{c}\text { Feld- } \\
\text { prüfung }\end{array}$ & $\begin{array}{c}\text { Prüf- } \\
\text { station }\end{array}$ & $\begin{array}{c}\text { Vertrags- } \\
\text { betriebe }\end{array}$ & Nukleus \\
\hline $\begin{array}{c}\text { Monetärer Gesamtzuchtfortschritt } \\
\text { mit Ultraschall } \\
\text { ohne Ultraschall }\end{array}$ & - & 1,00 & 1,16 & 1,05 & 1,76 \\
$\begin{array}{l}\text { Züchtungsertrag } \\
\text { mit Ultraschall } \\
\text { ohne Ultraschall }\end{array}$ & 1,08 & 0,74 & 1,00 & 0,75 & - \\
$\begin{array}{l}\text { Zulchtungskosten } \\
\text { mit Ultraschall } \\
\text { ohne Ultraschall }\end{array}$ & 9,18 & 8,68 & 10,03 & 9,27 & 13,01 \\
$\begin{array}{l}\text { Zilchtungsgewinn } \\
\text { mit Ultraschall } \\
\text { ohne Ultraschall }\end{array}$ & - & 5,86 & 7,70 & 6,08 & - \\
\hline
\end{tabular}

Allen in Tabelle 4 dargestellten Zuchtplänen ist gemeinsam, daß Zuchtpläne ohne Ultraschallmessungen kostenintensiver sind. Die Aufwendungen für die Durchführung von Ultraschallmessungen sind zwar etwas höher, durch den hohen Züchtungsertrag ist aber letztlich ein höherer Züchtungsgewinn zu erzielen.

Am lebenden Tier wird gegenwärtig die Schlachtkörperqualität mit einer Bemuskelungsnote oder mittels Ultraschallmessungen bewertet. Die Verwendung von Ultraschall in der Leistungsprüfung führt im Mittel der Zuchtpläne Feldprüfung, Prüfstation und Vertragsbetriebe zu einem jährlichen naturalen Zuchtfortschritt im Merkmal Fleischanteil von 0,55 \% (Tab. 5). Das ist im Durchschnitt der Zuchtpläne fast dreimal so viel im Vergleich zu diesen Zuchtplänen, in denen die Schlachtkörperqualität mit- 
tels der Bemuskelungsnote bewertet wird (Modelle ohne Ultraschallmessungen). Auch der monetäre jährliche Gesamtzuchtfortschritt ist bei den Varianten mit Ultraschallmessungen um 0,24 DM höher.

Tabelle 5

Vergleich von Zuchtplänen nach dem naturalen jährlichen Zuchtfortschritt im Merkmal Fleischanteil [\%] (Comparsion of breeding systems according to the annual monetary genetic gain for meat percentage)

\begin{tabular}{lccccc}
\hline & $\begin{array}{c}\text { Ausgangs- } \\
\text { situation }\end{array}$ & $\begin{array}{c}\text { Feld- } \\
\text { prüfung }\end{array}$ & $\begin{array}{c}\text { Prü- } \\
\text { station }\end{array}$ & $\begin{array}{c}\text { Vertrags- } \\
\text { betriebe }\end{array}$ & Nukleus \\
\hline $\begin{array}{l}\text { mit Ultraschall } \\
\text { ohne Ultraschall }\end{array}$ & - & 0,47 & 0,63 & 0,56 & 0,96 \\
\hline
\end{tabular}

In Tabelle 6 sind mit Ausnahme der Ausgangssituation nur Zuchtpläne mit Ultraschallmessungen dargestellt. Innerhalb eines optimierten Zuchtplanes leisten die einzelnen Zuchtzielmerkmale einen unterschiedlich hohen Beitrag zum Züchtungsertrag. Das Merkmal Aufgezogene Lämmer hat in der Feldprüfung einen Anteil am Züchtungsertrag von 17,6\%. Der höchste Anteil ist im Nukleus bedingt durch die Zweistufenselektion der Mütter nach diesem Merkmal erzielbar.

Tabelle 6

Vergleich der Zuchtpläne nach Züchtungserträgen [DM] (Comparsion of breeding systems according to their return)

\begin{tabular}{lccccc}
\hline Zielgrößen & $\begin{array}{c}\text { Ausgangs- } \\
\text { situation') }\end{array}$ & $\begin{array}{c}\text { Feld- } \\
\text { prüfung }\end{array}$ & $\begin{array}{c}\text { Prüf- } \\
\text { station }\end{array}$ & $\begin{array}{l}\text { Vertrags- } \\
\text { betriebe }\end{array}$ & Nukleus \\
\hline Aufgezogene Lämmer & 4,92 & 1,53 & 1,45 & 1,44 & 3,74 \\
Aufzuchtgewicht & 0,82 & 0,26 & 0,25 & 0,24 & 0,63 \\
Masttagszunahme & 2,54 & 1,79 & 1,74 & 1,42 & 1,85 \\
$\quad$ Fleischanteil & 0,90 & 5,10 & 6,59 & 6,17 & 6,79 \\
Züchtungsertrag & $\mathbf{9 , 1 8}$ & $\mathbf{8 , 6 8}$ & $\mathbf{1 0 , 0 3}$ & $\mathbf{9 , 2 7}$ & $\mathbf{1 3 , 0 1}$ \\
Anteil am Züchtungsertrag in [\%] & & & & & \\
$\quad$ Aufgezogene Lämmer & 53,6 & 17,6 & 14,4 & 15,5 & 28,7 \\
$\quad$ Aufzuchtgewicht & 8,9 & 2,9 & 2,4 & 2,6 & 4,8 \\
$\quad$ Masttagszunahme & 27,6 & 20,6 & 17,3 & 15,3 & 14,2 \\
$\quad$ Fleischanteil & 9,8 & 58,7 & 65,7 & 66,5 & 52,1 \\
\hline$\quad$ Zuchtplan ohne Ultraschallmessungen & & & & &
\end{tabular}

Das Aufzuchtgewicht hat mit einem Anteil zwischen 2,4 und 4,8 \% nur einen unwesentlichen Anteil. Das wichtigste Merkmal ist der Fleischanteil, dessen Anteil am Gesamtzuchtfortschritt zwischen 52,1 und 66,5\% liegt.

In der Regel wird es nicht immer möglich sein, die in Tabelle 4 dargestellten optimalen Zuchtpläne in der Praxis umzusetzen. Abweichungen vom Optimum sind aber gleichbedeutend mit Verlusten im Zuchtfortschritt bzw. im Züchtungsgewinn. Es soll deshalb in Tabelle 7 am Beispiel des Zuchtsystems mit Nukleus gezeigt werden, wie hoch die Veränderungen sind, wenn einzelne Populationsparameter partiell um eine Einheit vom Optimum abweichen. Daraus können Schlüsse gezogen werden, welche Populationsparameter möglichst wenig von Optimum abweichen sollten. 
Tabelle 7

Veränderung der Zielgrößen in [DM] bei partieller Variation verschiedener Populationsparametern und deren prozentuale Abweichung vom Optimum des Zuchtsystems Nukleus (Change of objective criteria [DM] when various parameters were partially varied; below the deviation from the optimum)

\begin{tabular}{|c|c|c|c|c|c|c|c|c|c|}
\hline Zielgrőßen & Optimum & 1 & 2 & 3 & 4 & 5 & 6 & 7 & 8 \\
\hline \multirow{2}{*}{$\begin{array}{l}\text { Mon. Zuchtfort. } \\
\text { Abweichung in [\%] }\end{array}$} & 1,76 & 1,76 & 1,58 & 1,55 & 1,71 & 1,76 & 1,71 & 1,76 & 1,73 \\
\hline & & 0 & $-10,2$ & $-11,9$ & $-2,8$ & 0 & $-2,8$ & 0 & $-1,7$ \\
\hline \multirow{2}{*}{$\begin{array}{l}\text { Züchtungsertrag } \\
\text { Abweichung in [\%] }\end{array}$} & 13,01 & 12,89 & 11,62 & 11,34 & 12,70 & 12,86 & 12,76 & 12,98 & 12,48 \\
\hline & & $-0,9$ & $-10,8$ & $-12,8$ & $-2,4$ & $-1,1$ & $-1,9$ & $-0,2$ & $-4,1$ \\
\hline \multirow{2}{*}{$\begin{array}{l}\text { Züchtungskosten } \\
\text { Abweichung in [\%] }\end{array}$} & 3,85 & 3,78 & 3,16 & 2,99 & 3,84 & 3,85 & 3,86 & 3,85 & 3,85 \\
\hline & & $-1,8$ & $-17,9$ & $-22,3$ & $-0,2$ & 0 & 0,2 & 0 & 0 \\
\hline \multirow{2}{*}{$\begin{array}{l}\text { Züchtungsgewinn } \\
\text { Abweichung in [\%] }\end{array}$} & 9,16 & 9,11 & 8,46 & 8,35 & 8,86 & 9,01 & 8,90 & 9,14 & 8,64 \\
\hline & & $-0,5$ & $-7,6$ & $-8,8$ & $-3,3$ & $-1,6$ & $-2,8$ & $-0,6$ & $-5,6$ \\
\hline $\begin{array}{ll}1 & \text { Prafgruppengröße } \\
2 & \text { Remontierungsanteil J } \\
3 & \text { Nutzungsdauer der Nu } \\
4 & \text { Genanteil der Jungscha } \\
5 & \text { Genanteil der Muttem } \\
6 & \text { Remontierungsanteil L } \\
7 & \text { Genanteil der Jungboc } \\
8 & \text { Nutzungsdauer der M }\end{array}$ & $\begin{array}{l}\text { Jungbðcke:Al } \\
\text { ukleusbðcke } \\
\text { hafe im Nukle } \\
\text { n aus Testherd } \\
\text { Lammer:Jung } \\
\text { cke in Produk } \\
\text { Mutterschafe in }\end{array}$ & $\begin{array}{l}\text { Joke im N } \\
\text { im Nukle } \\
\text { hafe in Tc } \\
\text { onsstufe } \\
\text { der Testhe }\end{array}$ & kleus & & & & & $\begin{array}{l}{[-1 \mathrm{Tie}} \\
{[-10 \%} \\
{[+1 \mathrm{Jah}} \\
{[+10 \%} \\
{[-10 \%} \\
{[+10 \%} \\
{[+10 \%} \\
{[+1] \text { ah }}\end{array}$ & \\
\hline
\end{tabular}

Verändert man verschiedenen Züchtungsparameter, so stellt sich heraus, daß die Populationsstruktur der Testherde einen wesentlichen Einfluß auf das Ergebnis hat. Verlängert man die Nutzungsdauer der Mutterschafe in der Testherde um ein Jahr, sinkt der Züchtungsgewinn bedingt durch den geringeren Züchtungsertrag um -5,6\% gegenüber dem Optimum (Pos. 8 in Tab. 7). Gleiches gilt für den Remontierungsanteil in der Testherde, nur vermindert sich der Züchtungsgewinn mit $-2,8 \%$ nicht in dem Maße. Den größten Einfluß auf den Züchtungsgewinn haben allerdings die Nutzungsdauer der Altböcke im Nukleus sowie der Remontierungsanteil der zweiten Selektionsstufe im männlichen Pfad. Werden die Altböcke im Nukleus ein Jahr länger genutzt bzw. wird der Remontierungsanteil um $10 \%$ vermindert, sinkt der Züchtungsgewinn um $-8,8 \%$ bzw. $-7,6 \%$ (Pos. 2 und 3 in Tab. 7). Die Abweichungen dieser beiden Populationsparameter vom Optimum beeinflussen auch am stärksten den monetären jährlichen Gesamtzuchtfortschritt.

3.

\section{Diskussion}

Das Merinofleischschaf in den ostdeutschen Bundesländern ist zu einer Rasse im Zwei-Nutzungs-Typ Fleisch-Wolle umzuzüchten. Während das bisherige Zuchtziel in der Verbesserung der Wolleistung bestand, erfordert das heutige Preisgefüge die Betonung der Mast- und Schlachtleistung.

Das Zuchtziel sollte die Merkmale „Aufgezogene Lämmer je Mutterschaf und Ablammung“, „Summe der Absetzgewichte der Lämmer“, die Masttagszunahme und den Fleischanteil umfassen. Nach den Ergebnissen der Planungsrechnungen leisten die einzelnen Merkmale einen unterschiedlich hohen Beitrag zum Züchtungsertrag. Den höchsten Anteil am Züchtungsertrag im Mittel aller Zuchtpläne mit Ultraschall (Feld- 
prüfung, Prüfstation, Vertragsbetriebe und Nukleus) hat das Merkmal „Fleischanteil“ mit $60,7 \%$. Es folgen die Merkmale „Aufgezogene Lämmer je Mutterschaf und Ablammung“ mit 19,0\%, die Masttagszunahme mit 16,8\% und das Merkmal „Summe der Absetzgewichte der Lämmer“ mit 3,2\%. Mit geringfügigen Schwankungen sind diese Relationen bei allen untersuchten Zuchtplänen wiederzufinden. Für den monetären Gesamtzuchtfortschritt hat das Merkmal „Summe der Aufzuchtgewichte der Lämmer" nur eine untergeordnete Bedeutung. Gemessen am Nutzen ist der Aufwand zur Erfassung dieses Merkmals recht hoch. Es wäre deshalb zu prüfen, inwieweit die Aufzuchtleistung eines Mutterschafes mit einem anderen Merkmal nicht besser bewertet werden kann.

In der vorliegenden Arbeit wurden Zuchtprogramme untersucht, die sich hauptsächlich in der Art der Leistungsprüfung, in der Populationsstruktur und in den Übertragungswegen des Zuchtfortschritts unterscheiden. Auch wenn MOET-Zuchtprogramme prinzipiell möglich sind (HECKENBERGER, 1991, NIEBEL und FEWSON, 1978), ist gegenwärtig eine praktische Durchführbarkeit mit einem zu hohen Aufwand verbunden. Während eine multiple Ovulation problemlos ausgelöst werden kann, ist eine transzervikale Spülung aufgrund des Gebärmutteraufbaus fast nicht möglich und eine Embryonengewinnung durch Laparoskopie sehr aufwendig. Auch bei Zuchtprogrammen mit künstlicher Besamung müssen Abstriche gemacht werden, da die Befruchtungsfähigkeit von Frischsperma recht schnell nachläßt und die Konzeptionsrate bei Besamung von Tiefgefriersperma mit $30 \%$ bis $40 \%$ vergleichsweise gering ist. Hinzu kommt der hohe Arbeitskräftebedarf bei diesem Verfahren.

Vergleicht man die fünf vorgestellten Prüfsysteme, so fällt auf, daß bereits die Ausgangssituation mit 7,19 DM einen sehr hohen Züchtungsgewinn hervorbringt. Ein vergleichbarer Züchtungsgewinn wird nur noch mit dem Zuchtplan „Vertragsbetriebe“ erzielt, bei dem in der Leistungsprüfung stationsähnliche Bedingungen unterstellt werden. Das heißt, Zuchtpläne mit einer Nachkommenprüfung im Feld (Zuchtplan „Feldprüfung“) und Zuchtpläne mit einer Prüfstation sind im Vergleich zur Ausgangssituation zu teuer, um den Aufwand lohnend erscheinen zu lassen.

Werden die einzelnen Prüfsysteme auf der Basis des monetären jährlichen Gesamtzuchtfortschritts verglichen, dann sind Prüfsysteme mit einer Stationsprüfung der Feldprüfung vorzuziehen. Der höhere Zuchtfortschritt ergibt sich aus der exakteren Leistungsprüfung. Ähnliche Ergebnisse erhielt KALM (1976) bei Planungsrechnungen in einer Schweinepopulation. Danach wurden mit der Eigenleistung im Feld die höchsten Züchtungsgewinne, mit der Eigenleistungsprüfung auf Station jedoch die höheren Zuchtfortschritte je Tier erzielt. Nachteilig wirkt sich die in der Regel begrenzte Prüfkapazität aus. Dieses Problem kann umgangen werden, wenn die Leistungsprüfung in kontrollierte Betriebe verlagert wird. Diese Variante untersuchten NIEBEL und FEWSON (1978). Sie fanden heraus, daß die Nachkommenprüfung der Bullen auf Fleischleistung in kontrollierten Mastbetrieben der Eigenleistungsprüfung der Jungbullen auf Station im Zuchtfortschritt um $5 \%$ und im Züchtungsgewinn um $3 \%$ überlegen war. Auch in den vorliegenden Untersuchungen war der Züchtungsgewinn und der monetäre Gesamtzuchtfortschritt des Zuchtplanes „Vertragsbetriebe“ mit Ultra- 
schall dem der Feldprüfung um $5 \%$ überlegen.

Der Zuchtplan mit einer Prüfstation ist vergleichsweise teuer. Auch wenn bei diesem Zuchtplan ein etwas höherer monetärer jährlicher Gesamtzuchtfortschritt zu erwarten ist, so stellt erst ein Zuchtsystem mit einem Nukleus eine echte Alternative dar, weil mit diesem Zuchtsystem sowohl der höchste Züchtungsgewinn als auch der höchste Zuchtfortschritt erzielt werden kann. Im Zuchtplan „Prüfstation“ beträgt der monetäre jährliche Gesamtzuchtfortschritt 1,16 DM. Das sind 0,16 DM mehr als im Zuchtplan „Feldprüfung". Da diese Differenz nur sehr gering ist und der Züchtungsgewinn vergleichsweise niedrig ausfällt, sollte in weiterführenden Untersuchungen überprüft werden, ob auf eine Prüfstation in der Leistungsprüfung beim Schaf verzichtet werden könnte.

Der Vergleich der verschiedenen Zuchtsysteme hinsichtlich der Übertragungswege des Zuchtfortschritts bzw. der Populationsstruktur führte zu folgenden Ergebnissen:

- In der Feldprüfung schneiden Zuchtpläne mit einer Zweistufenselektion der männlichen Tiere gegenüber Zuchtplänen mit einer ausschließlichen Eigenleistungsprüfung bezogen auf den Züchtungsgewinn schlechter ab. Auch der monetäre jährliche Gesamtzuchtfortschritt ist etwas geringer, weil durch die Einführung der Nachkommenprüfung die Mutterschafe in der Zuchtstufe geteilt werden müssen für Anpaarungen mit Altböcken und für Testpaarungen mit Jungböcken. Im Vergleich zur Ausgangssituation, in der die Leistungsprüfung im Feld nur als eine Einstufenselektion durchgeführt wird, zieht diese Teilung ein längeres Generationsintervall und eine geringere Selektionsintensität im männlichen Pfad nach sich. Diese beiden Faktoren bewirken, daß die Vorteile der Nachkommenschaftsprüfung und damit der Zweistufenselektion nicht zur Wirkung kommen. Diese Feststellung trifft in noch stärkerem Maße zu, wenn keine Jungböcke in der Zuchtstufe eingesetzt werden.

- Bei KOMINAKIS (1991) wird mit einem Nukleus der höchste Zuchtfortschritt erzielt. Er führt das auf die genauere Selektion im Nukleus zurück, während der Öffnungsgrad des Nukleus seiner Meinung nach keine Rolle spielt. Im Gegensatz dazu wird in Übereinstimmung mit COLLEAU (1986) die in dieser Arbeit aufgezeigte Überlegenheit des Nukleus auf die Einführung einer Teststufe und der damit verbundenen Öffnung des Nukleus zurückgeführt, weil dadurch sowohl für den Nukleus als auch für die Testherde eine breitere Selektionsbasis zur Verfügung steht. Der optimale Öffnungsgrad des Nukleus beträgt bei Optimierung nach Züchtungsgewinn $30 \%$, während zur Erzielung eines hohen Zuchtfortschritts ein Öffnungsgrad des Nukleus von $50 \%$ günstiger ist. Ein derartiger Zuchtplan stellt allerdings sehr hohe Anforderungen an die Organisation der Zuchtarbeit. Es erscheint deshalb unter den gegenwärtig gegebenen praktischen Bedingungen der Schafzucht in Deutschland noch unrealistisch, dieses Zuchtsystem umzusetzen.

- Sehr hoch ist der Einfluß der Ultraschallmessungen auf den Zuchtfortschritt. Zuchtpläne mit Ultraschall haben einen um 0,23 DM höheren monetären jährlichen Gesamtzuchtfortschritt. Die Verwendung von Ultraschall in der Leistungsprüfung führt im Mittel aller Zuchtpläne zu einem jährlichen naturalen Zuchtfortschritt im Merkmal Fleischanteil von 0,553\%. Das sind durchschnittlich fast $300 \%$ mehr im 
Vergleich zu Zuchtplänen, in denen die Schlachtkörperqualität mittels der Bemuskelungsnote bewertet wird. Die Aufwendungen für die Durchführung von Ultraschallmessungen sind zwar etwas höher, durch den hohen Züchtungsertrag ist aber letztlich ein höherer Züchtungsgewinn zu erzielen. Gegenüber der bisherigen Verfahrensweise besteht der Vorteil der Ultraschallmessungen vor allem darin, eine hinreichend genaue Aussage zum Fleischanteil bereits am lebenden Tier machen zu können.

Unter Berücksichtigung der in dieser Arbeit erzielten Ergebnisse und der Durchführbarkeit in der praktischen Schafzucht erscheint ein Zuchtsystem am zweckmäßigsten, in dem männliche Lämmer in einem vertraglich gebundenen Betrieb unter stationsähnlichen Bedingungen bei Verzicht auf eine Prüfstation zentral aufgezogen werden. Eine Einstufenselektion bei weiblichen wie männlichen Tieren erscheint ausreichend. Aufgrund der hohen Aufwendungen und Kosten sollte auf eine Schlachtung verzichtet werden und statt dessen die Schlachtkörperqualität in der Eigenleistungsprüfung sowohl an weiblichen als auch an männlichen Tieren mittels Ultraschall beurteilt werden.

\section{Literatur}

BICHARD, M.:

Dissemination of genetic improvement through a livestock industry. Anim. Prod., Edinburgh 13 (1971), 401-411

BRASCAMP, E.W.:

Deterministic model and monte carlo method. In: Methods on economic optimisation of animal breeding plans, Rapport 134 (1978), 11-15

COLLEAU, J.J.:

Genetic improvement by embryotransfer within an open selection nucleus in dairy cattle. Proc. 3rd World Cong. Gen. Appl. Livest. Prod. XII, (1986), 127-132

ELSEN, J.M.; MOCQUOT, J.C.:

Récherches pour une rationalisation technique et econimique des schémas de selection des bovins et ovins. II. Methode du prevision de l'évolution du niveau genetique d'une population soumise a une operation de selection des bovins et ovins. Bull. Technique. Dept. Genet. Anim. 17 (1974), 30-54

HECKENBERGER, G.:

Planungsrechnungen über den Einfluß von Grenznutzenwerten der Leistungsmerkmale, Parametern der Populationsstruktur und von Züchtungssystemen auf den Züchtungserfolg beim Zweinutzungsrind.

HILL, G.W.: Univ. Hohenheim, Diss., 1991

Prediction and evaluation of response to selection with overlapping generations. Anim. Prod., Edinburgh 18 (1974), 117-139

KALM, E.:

Ökonomischer Vergleich von genetischen Prüfungsalternativen in Schweinezuchtprogrammen. 27. Jahrestagung der EVT, Zürich, 1976

KARRAS, K.; NIEBEL, E.; NITTER, G.; BARTENSCHLAGER, H.:

User`s guide for ZPLAN. Univ. Hohenheim, 1994

KOMINAKIS, A.P.:

Model calculations for optimizing breeding schemes in the Karagouniko dairy sheep of Greece. Univ. Hohenheim, Diss., 1991

MỨLLER, U.:

Planungsrechnungen zur Optimierung von Zuchtsystemen für die Verbesserung der Mastleistung und Schlachtkörperqualität der Rasse Merinofleischschaf unter Verwendung des Ultraschallmeßverfahrens am lebenden Tier. Univ. Leipzig, Diss., 1995 


\section{NIEBEL, E.; FEWSON, D.:}

Untersuchungen zur Zuchtplanung für die Reinzucht beim Zweinutzungsrind - 4. Auswahl der PrüWEBER, F.: fungsmethoden für die Zuchtwahl auf Fleischleistung. Züchtungskunde, Stuttgart 50 (1978), 264-275

Selektionsalternativen bei Kühen und ihr Einfluß auf den Altersaufbau der Bestände, den genetischen Trend und den Herdendurchschnitt. Z. Tierzüchtung Züchtungsbiol., Berlin, Hamburg 93 (1976), 156168

Eingegangen: 17.07 .1998

Akzeptient: 02.03.1999

Anschriften der Verfasser

Dr. ULF MƯLLER

Sächsische Landesanstalt für Landwirtschaft

Am Park 3

D-04860 Köllitsch

Dr. KNUT STRITTMATTER

Sächsische Landesanstalt für Landwirtschaft

Bornaische Str. 31/33

D-04416 Markkleeberg

Dr. GERHARD NITTER

Universität Hohenheim

Institut für Tierhaltung und Tierzüchtung

Garbenstraße 17

D-70599 Stuttgart 


\title{
Buchbesprechung
}

\author{
Auf Haustierspuren zu den Ursprüngen der Japaner - Vor- und Frühgeschichtliche Haustierhaltung in
Japan
}

\section{ULRIKE THIEDE}

152 Seiten, 55 Abbildungen, kt, Indicium Verlag München, 1998, ISBN 3-89129-429-8, 38,00 DM

Wohl jedes Volk befaßt sich mit dem Problem der Herkunft seiner Vorfahren. Wann kamen sie? Woher kamen sie? Diese Fragen können auf unterschiedliche Weise beantwortet werden: Durch Ausgrabungsfunde, durch Ähnlichkeiten mit anderen Ethnien sowie durch Nutzpflanzen und insbesondere Haustiere. Zugegeben: Verwandtschaften zwischen Haustieren sagen nicht notwendigerweise etwas über den Wanderweg der Vorfahren aus. Es ist in gleicher Weise denkbar, daß Tiere von Volk zu Volk weitergegeben wurden. Doch diese Frage interessiert den Tierzüchter vermutlich weniger.

Über die Haustiere Japans ist in Mitteleuropa wenig bekannt. Das vorliegende Buch ist eine umfangreiche und sorgfältige Analyse der relevanten Literatur. Es stellt die einzelnen domestizierten Tierarten vor und befaßt sich auch mit den Gründen für deren Import nach Japan. So erscheinen Rinder erstmals in der Yayoi-Zeit (300 v. Chr. bis $300 \mathrm{n}$. Chr.), praktisch gleichzeitig mit der Einführung der Naßreiskultivienung. Vielleicht würde man in diesem Zusammenhang Wasserbüffel erwarten, aber diese Tierart ist nur für tropisches und subtropisches Klima geeignet. Zumindest auf der Hauptinsel Honschu und weiter nördlich ist es für sie zu kalt. Pferde kamen offenbar mit Reitervölkern aus Zentralasien nach Japan. Ziegen und Schafe haben in der frühen Geschichte dieses Landes keine Bedeutung. Sie wurden erst seit dem 19. Jahrhundert durch die Europäer nach Japan eingeführt.

Einschneidenden Einfluß auf die japanische Einstellung zum Tier hatte der von China und Korea übernommene Buddhismus, der 538 n. Chr. zur Staatsreligion erklärt wurde. Damit war ein Verbot des Tötens von Tieren und des Verzehrs von Fleisch verbunden. Das traf vor allem Arten, die ausschließlich für den Fleischverzehr gehalten wurden, wie das Schwein. Für den an Haustierrassen Interessierten sind die zahlreichen Dendrogramme, welche die verwandtschaftlichen Beziehungen der einzelnen Rassen innerhalb einer Art wiedergeben, sehr aufschlußreich. Für viele japanische Nutztierrassen liegen differenzierte Beschreibungen vor.

Das Buch ist konzentriert und dennoch gut lesbar geschrieben. Abbildungen und Tabellen geben einen guten Überblick über das doch ziemlich verwickelte Geschehen. Das Werk wird alle die fesseln, die an der Geschichte der Haustiere und ihrer Rassen interessiert sind. 